\title{
Correlation of maternal-fetal attachment and health practices during pregnancy with neonatal outcomes
}

\author{
Maryam Sadat Maddahi ${ }^{1}$, Mahrokh Dolatian ${ }^{2}$, Monirsadat khoramabadi ${ }^{1}$, Atefeh Talebi ${ }^{3}$
}

\begin{abstract}
${ }^{1}$ M.Sc. of Midwifery, Department of Midwifery and Reproductive Health, School of Nursing and Midwifery, Shahid Beheshti University of Medical Sciences, Tehran, Iran

${ }^{2} \mathrm{Ph} . D$. of Social Determinants of Health, Assistant Professor, Department of Midwifery and Reproductive Health, School of Nursing and Midwifery, Shahid Beheshti University of Medical Sciences, Tehran, Iran

${ }^{3}$ Ph.D., Department of Biostatistics, Shahid Beheshti University of Medical Sciences, Tehran, Iran
\end{abstract}

\section{Type of article: Original}

\begin{abstract}
Introduction: Low birth weight due to preterm delivery or intrauterine growth restriction (IUGR) is the strongest factor contributing to prenatal, neonatal, and postnatal mortality. Maternal-fetal attachment plays a significant role in maternal and fetal health. Health practices performed by the mother during pregnancy constitute one of the factors that may affect neonatal outcomes. The present study was conducted to identify the relationship between maternal-fetal attachment and health practices during pregnancy with neonatal outcomes.

Methods: This cross-sectional study was conducted on 315 pregnant women with a gestational age of 33-41 weeks who presented to hospitals in Sirjan (Iran) between December 2014 and February 2015. The data collection tools used included the Health Practices in Pregnancy Questionnaire and the Maternal Fetal Attachment Scale. Data were analyzed using IBM-SPSS version 20, focusing on the Pearson product-moment correlation and the logistic regression model. Statistical significance was set to $\mathrm{p}<0.05$.

Results: The mean score of maternal-fetal attachment was 60.34, and the mean score of health practices was 123.57. The mean birth weight of the neonates was $3052.38 \mathrm{~g}$. Health practices $(\mathrm{p}<0.05, \mathrm{r}=0.11)$ and maternalfetal attachment $(\mathrm{p}<0.01, \mathrm{r}=0.23)$ were positively and significantly correlated with neonatal outcomes. A significant positive relationship was also observed between maternal-fetal attachment and neonatal outcomes. No significant relationships were observed between health practices during pregnancy and neonatal outcomes.

Conclusion: Maternal-fetal attachment and health practices during pregnancy are positively and significantly correlated with neonatal outcomes.
\end{abstract}

Keywords: Maternal-fetal attachment, Health practices; Pregnancy, Neonatal outcomes

\section{Introduction}

Low birth weight due to a preterm delivery or intrauterine growth restriction (IUGR) is the strongest factor contributing to prenatal, neonatal, and postnatal mortality as well as infant mortality (1). Prenatal mortality is a major epidemiological indicator of maternal and infant health. Inadequate maternal care may lead to prenatal mortality (2). Neonatal mortality is 40 times higher in neonates with a low birth weight (LBW) than those with a normal birth weight; LBW neonates are also significantly prone to short- or long-term diseases, such as cognitive developmental delay, delayed growth, abdominal problems, and respiratory diseases as well as the risk of obesity (3). The lower the birth weight or the shorter the duration of pregnancy, the higher the neonate mortality rate (4). Low socioeconomic statuses, poor nutrition, smoking, consumption of alcohol or drugs, a history of low birth weight, anemia, maternal age below 18 or over 35, birth order, short stature and low weight at the beginning of pregnancy are risk factors of low birth weight (5). Attachment is a stable emotional bond between two individuals (6). Maternal-fetal attachment involves behaviors and actions that indicate the mother's emotional bond with the

\section{Corresponding author:}

Assistant Professor Dr. Mahrokh Dolatian, Department of Midwifery and Reproductive Health, School of Nursing and Midwifery, Shahid Beheshti University of Medical Sciences, Tehran, Iran.

Tel: +98.2188202517, Fax: +98.2188202517, Email: mhdolatian@gmail.com

Received: March 05, 2016, Accepted: June 01, 2016, Published: July 2016

iThenticate screening: May 28, 2016, English editing: June 21, 2016, Quality control: July 04, 2016

(C) 2016 The Authors. This is an open access article under the terms of the Creative Commons Attribution-NonCommercialNoDerivs License, which permits use and distribution in any medium, provided the original work is properly cited, the use is non-commercial and no modifications or adaptations are made. 
fetus. These behaviors cause the early start and continuation of parental care, proper nutrition, sleep and exercise, abstinence from alcohol and drugs, and the desire to get to know the fetus during pregnancy, which ultimately result in a desirable pregnancy outcome and promote maternal and neonatal health (7). These behaviors also play a key role in parental identity formation, the future relationship between the mother and the neonate, and the infant's growth and development (8). Pillitteri concluded that this attachment, which is crucial for a successful maternal adaptation to pregnancy, develops gradually from the beginning of pregnancy and reaches its peak in the third trimester as well as continuing after delivery. A wide range of attachment behaviors emerge during pregnancy, such as talking to the fetus and touching and caressing the belly. This attachment may begin with pregnancy or during the first ultrasound or when feeling the first movement of the fetus and may then improve gradually with gestational age and with the increase in fetal movements (9). Maternal-fetal attachment plays a key role in both the maternal and fetal health (10), prolongs the pregnancy, and affects the child's future social-emotional and cognitive relationships (11). Women who experience higher levels of maternal-fetal attachment present better self-care behaviors during pregnancy and perform better health practices. In contrast, women with low attachment levels are more likely to give birth to babies with poor health conditions due to their lower compliance with health practices during pregnancy (3). Various factors affect maternal-fetal attachment, such as communication within the family, pregnancy acceptance and support, the mother's mental self-image, a previous history of pregnancy, obstetric and medical complications during pregnancy and the pregnancy being unwanted (12). Increasing maternal-fetal attachment encourages the mother to perform healthy behaviors during pregnancy, such as smoking and alcohol withdrawal, proper nutrition, exercise, ongoing pregnancy care, education about the fetus, and participation in childbirth preparation classes, which cause a satisfactory pregnancy outcome and promote the mother's and child's health. The health practices in which the pregnant mother is involved may affect neonatal outcomes (3). Positive health practices include the lack of addiction, consistent care, good nutrition, a proper diet, sufficient rest and sleep, participation in consistent exercises, and education about pregnancy and childbirth. Several variables are correlated with health practices during pregnancy, including the socioeconomic status and the degree of education and high social support (3). According to articles 3 and 4 of the Regulations for Midwives approved by the Iranian Ministry of Health and Medical Education, preparing pregnant women for the maternal role is one of the major responsibilities of a midwife; nevertheless, in Iran, the greatest part of prenatal services is still limited to physical maternal care while the psychological needs of pregnant women are less emphasized (13). Attachment behaviors appear to be different from one culture to another, as every culture has its own belief system with respect to major life events, such as pregnancy and childbirth; providing healthcare requires an adequate knowledge of this belief system. A religious or cultural background may strongly influence the way the mother assumes her pregnancy role, and some beliefs and superstitions may also impose limitations on the individual's behaviors. Alhusen emphasized the effect of a society's culture on maternal-fetal attachment behaviors and encouraged further studies on this subject, especially in developing countries (10). Given the limited number of studies on this subject in Iran, the present study seeks to examine the correlation of maternal-fetal attachment and health practices during pregnancy with neonatal outcomes.

\section{Material and Methods \\ 2.1. Design and setting}

The present cross-sectional study was conducted on a study population consisting of pregnant women with a gestational age of 33-41 weeks presenting to hospitals in Sirjan for delivery between December 2014 and February 2015. The study sample size was estimated at 315 , and two hospitals in Sirjan were sampled through continuous convenience sampling.

\subsection{Inclusion and exclusion criteria}

The inclusion criteria consisted of a minimum age of 19, the pregnancy being intended, no known diseases, no known abnormalities of the fetus, and a gestational age of 33-41 weeks. Exclusion criteria consisted of those who did not answer all of the questions on the questionnaire and those who exited the study.

\subsection{Measurement tools}

Three questionnaires were used to collect the data. A researcher-made demographic questionnaire consisting of four items was used to assess the study population's personal details, including age, degree of education, income, marital status, the first day of the last menstrual cycle, gravidity, parity, the number of abortions, and the history of stillbirths. Cranley's 24-item Maternal Fetal Attachment Scale of 1981 was used to assess maternal-fetal attachment within five subscales, including differentiation of the self from the fetus, interaction with fetus, attribution of characteristics to the fetus, giving of the self, and role taking. Respondents rated the scale items on a three-point 
Likert scale ranging from 1 (definitely no) to 3 (definitely yes), with items 14 and 22 being reverse-scored. The scores ranged from 24 to 72 , and higher scores indicated a higher level of maternal-fetal attachment. Cranley confirmed the internal reliability of the questionnaire with a Cronbach's alpha of 0.85 ; however, the present study calculated the scale's Cronbach's alpha to be 0.86 (14). In 2003, Shakespear designed the Health Practices in Pregnancy Questionnaire with 34 items and six subscales, including a balance of work and rest, safety measures, nutrition, avoidance of drug use, receipt of adequate healthcare, and receipt of accurate information about delivery. Respondents rated the items on a five-point Likert scale ranging from 1 (never) to 5 (always), with items 4, 5, 6, 7, $11,19,20,21$, and 31 being reverse-scored. The scores ranged from 34 to 170 . Scores closer to 170 indicated better health practices. The present study calculated a Cronbach's alpha of 0.83 for this questionnaire (15).

\subsection{Data collection and analysis}

After confirming the validity and reliability of the data collection tools, obtaining an introduction letter from Shahid Beheshti School of Nursing and Midwifery, and obtaining the permission of Sirjan University of Medical Sciences, sampling started in Imam Reza Hospital and Gharazi Hospital. After identifying the eligible candidates, the study samples were briefed on the study objectives in person and were then ensured of the confidentiality of their information. After submitting their consent form, participants completed the questionnaires individually. The data obtained from the questionnaires were then analyzed using IBM SPSS Statistics version 20 (IBM Corp., Armonk, NY, USA). Descriptive statistics and inferential statistics were used to analyze the data and establish relationships among the variables. The Pearson product-moment correlation's coefficient was used to investigate the correlation between the variables, and regression models were used to examine the relationships between the independent and dependent variables.

\section{Results}

The participants were ranged in age from 19 to 50 and had a mean age of 27.63; their mean gestational age was also 27.6 weeks. The majority of the participants had a high school diploma and an income between 5,000,000 and $10,000,000$ IRR. The majority of the participants had a gestational age of 39 weeks and was experiencing their first pregnancy. According to the data, participants' maternal-fetal attachment scores ranged from 24 to 72 and had a mean of 60.34; the health practices during pregnancy scores obtained ranged from 53 to 156 and had a mean of 123.57. The mean weight of the newborns ranged from 500 to $4000 \mathrm{~g}$ and had a mean of $3052.38 \mathrm{~g}$. (Table 1).

Table 1. Demographic and pregnancy details

\begin{tabular}{|c|c|c|c|c|c|}
\hline \multicolumn{2}{|c|}{ Demographic and pregnancy details } & $\mathrm{n}$ & $\%$ & Mean & SD \\
\hline \multirow[t]{4}{*}{ Age (year) } & $19-25$ & 112 & 35.6 & \multirow[t]{4}{*}{27.63} & \multirow[t]{4}{*}{4.85} \\
\hline & $26-30$ & 128 & 40.6 & & \\
\hline & $31-40$ & 73 & 23.2 & & \\
\hline & $41-50$ & 2 & 0.6 & & \\
\hline \multirow[t]{3}{*}{ Education } & Less than high school & 68 & 21.6 & \multirow[t]{3}{*}{-} & \multirow[t]{3}{*}{-} \\
\hline & High school diploma & 161 & 51.1 & & \\
\hline & College & 86 & 27.3 & & \\
\hline \multirow[t]{2}{*}{ Marital Status } & Married & 313 & 99.4 & \multirow[t]{2}{*}{-} & \multirow[t]{2}{*}{-} \\
\hline & Widow & 2 & 0.6 & & \\
\hline \multirow[t]{3}{*}{ Income (IRR) } & $\leq 5,000,000$ & 53 & 16.8 & \multirow[t]{3}{*}{2.1} & \multirow[t]{3}{*}{0.7} \\
\hline & $5,000,000-10,000,000$ & 149 & 47.3 & & \\
\hline & $\geq 10,000,000$ & 113 & 35.9 & & \\
\hline \multirow[t]{2}{*}{ Gestational age (weeks) } & $33-36$ & 15 & 4.7 & \multirow[t]{2}{*}{27.6} & \multirow[t]{2}{*}{4.84} \\
\hline & $37-41$ & 300 & 95.3 & & \\
\hline \multirow[t]{4}{*}{ Parity } & 1 & 107 & 34 & \multirow[t]{4}{*}{2.18} & \multirow[t]{4}{*}{1.13} \\
\hline & 2 & 94 & 29.8 & & \\
\hline & 3 & 76 & 24.1 & & \\
\hline & $\leq 4$ & 38 & 12.1 & & \\
\hline \multirow[t]{5}{*}{ Number of children } & 0 & 127 & 40.3 & \multirow[t]{5}{*}{-} & \multirow[t]{5}{*}{-} \\
\hline & 1 & 103 & 32.7 & & \\
\hline & 2 & 67 & 21.2 & & \\
\hline & 3 & 15 & 4.8 & & \\
\hline & 4 & 3 & 1 & & \\
\hline
\end{tabular}


The Pearson product-moment correlation coefficient obtained revealed health practices $(\mathrm{p}<0.05, \mathrm{r}=0.11)$ and maternal-fetal attachment $(\mathrm{p}<0.01, \mathrm{r}=0.23)$ to be significantly and positively correlated with neonatal outcomes $(\mathrm{p}<0.01, \mathrm{r}=0.23)$. All the attachment components examined were found to be significantly and positively correlated with neonatal outcomes except for giving of self $(\mathrm{r}=0.07)$. No significant positive relationships were found between health practices and neonatal outcomes. However, safety $(\mathrm{r}=0.11)$ and maternal-fetal attachment $(\mathrm{r}=0.23)$ were found to be significantly and positively correlated with neonatal outcomes $(\mathrm{p}<0.01)$. Maternal-fetal attachment $(\mathrm{p}<0.0001, \beta=0.22)$ predicted birth weight and explained $0.08 \%$ of its total variance, while health practices $(\mathrm{p}<0.13$, $\beta=0.08)$ did not predict birth weight.

\section{Discussion}

The results obtained showed good levels of maternal-fetal attachment and health practices during pregnancy among participating mothers. Health practices and maternal-fetal attachment were found to be significantly and positively correlated with neonatal outcomes, which is consistent with the results obtained by Alhusen et al. (3). Lindgren concluded that maternal-fetal attachment behaviors cause the early start and continuation of pregnancy care, proper nutrition, sufficient sleep and exercise, and abstinence from alcohol and drugs, which ultimately lead to desirable pregnancy outcomes and promote both the maternal and fetal health (7). All the components of attachment had a significant and positive correlation with neonatal outcomes, which is consistent with the results obtained by Alhusen et al. (3), who reported the mean score of attachment as 84.1 in their study entitled "The influence of maternal-fetal attachment and health practices on neonatal outcomes in low-income urban women." They found that most participants had medium attachment. The subscales for giving of the self and role taking received the highest scores while the subscales of attributing characteristics to the fetus and interaction with the fetus received the lowest scores, which is consistent with the results obtained by Chanachote (16). The low scores obtained in the latter two subscales may be attributed to how behaviors contributing to interaction with the fetus, including talking to the fetus as well as reading poetry and stories to the fetus, occur when the fetus is perceived by the parents as an independent creature capable of thinking and hearing. Other factors that may have contributed to the low scores include the mother's fear of harming the baby or her shame of performing these behaviors. The only component that was found not to be significantly and positively correlated with neonatal outcomes was self-sacrifice. In our study no significant relationships were observed between health practices and neonatal outcomes, although, in 2013, Alhusen et al. found a positive correlation between the two variables. The disparity of findings can be attributed to differences in culture, beliefs, socioeconomic status, and study settings. The correlation of maternal-fetal attachment and the subscales of health practices during pregnancy with neonatal outcomes indicates that safety and maternal-fetal attachment $(r=0.23)$ are significantly and positively correlated with neonatal outcomes $(p<0.01)$, which is consistent with the results obtained by Alhusen et al. In the present study, no significant relationships were observed among the balance of rest and work, maternal-fetal attachment, and birth weight. The majority of the women examined in this study (42.5\%) slept for at least eight hours a day at times. According to the data, $97.5 \%$ of the participants did not smoke or use drugs and 99.4\% did not drink. Fogarasi-Grenczer et al. (17) and Castell et al. (5) found that recreational drug use, smoking, drinking, and the use of other drugs increase the risk of low birth weight. In $50 \%$ of the cases, addicted pregnant women give birth to LBW infants, which is not necessarily caused by preterm delivery; this finding is consistent with the results obtained in the present study, as $92.4 \%$ of the women examined in this study gave birth to babies weighing $2501-4000 \mathrm{~g}$. The majority of the participants $(89 \%)$ maintained proper nutrition and used supplements for pregnancy. The mother's nutrition, especially in the third trimester, and lack of supplementation are factors that contribute to low birth weight. Nutrition factors are associated with low birth weight in preterm babies only (17). A significant relationship was observed between iron deficiency and preterm delivery (18). Maternal weight gain during pregnancy is also a factor that affects birth weight and preterm delivery (18). The shortage or lack of prenatal care is a risk factor for low birth weight as well as preterm delivery (19) Problems such as low birth weight and neonatal mortality are more common in women who have received insufficient prenatal care (20). The majority of the participants $(54.3 \%)$ had been to all their prenatal care visits, which is consistent with the results of the cited studies. The majority of the participants also followed a good dental hygiene routine. Low weight gain during pregnancy is associated with preterm delivery and low birth weight (19). The majority of the participants $(73 \%)$ made an effort to reach their healthiest weight. As every culture has its own belief system with respect to major life events such as pregnancy and childbirth, providing healthcare requires an adequate knowledge of this system. The present study failed to take into account participants' religious and cultural background. 


\section{Conclusions}

Our findings revealed the correlation of health practices and maternal-fetal attachment with neonatal outcomes. The majority of the subjects had high maternal-fetal attachment and good levels of health practices during pregnancy. All attachment components had a significantly positive correlation with neonatal outcomes. The only component that was found not significantly and positively correlated with neonatal outcomes was self-sacrifice. Similarly, no significantly positive relationships were observed between health practices and neonatal outcomes; however, safety was found to be significantly and positively correlated with neonatal outcomes. Given the complications of low birth weight, identifying the factors contributing to low birth weight and taking preventive measures are very important steps. No definite measures are currently taken to identify high risk pregnancies and low birth weight. The greatest part of prenatal services in Iran is limited to physical maternal care while the psychological needs of pregnant women are less addressed. Low birth weight can be prevented by identifying pregnant women with low levels of maternal-fetal attachment and health practices during pregnancy. The promotion of physical and mental health during pregnancy has become a target in the new millennium. Good feelings in pregnant women were also found to be associated with fewer complications during pregnancy and better neonatal outcomes.

\section{Acknowledgments:}

This research was derived from a master's thesis in the field of midwifery. The authors would like to express their gratitude to all the colleagues and participants who helped conduct this study, especially the authorities of Shahid Beheshti University of Medical Sciences, Gharazi Hospital, and Imam Reza Hospital.

\section{Conflict of Interest:}

There is no conflict of interest to be declared.

\section{Authors' contributions:}

All authors contributed to this project and article equally. All authors read and approved the final manuscript.

\section{References:}

1) Nobile CG, Raffaele G, Altomare C, Pavia M. Influence of maternal and social factors as predictors of low birth weight in Italy. BMC public health. 2007; 7: 192. doi: 10.1186/1471-2458-7-192. PMID: 17683559, PMCID: PMC1959188.

2) Siddalingappa H, Murthy MRN, Kulkarni P, N C A. Prevalence and factors influencing perinatal mortality in rural Mysore, India. J Clin Diagn Res. 2013; 7(12): 2796-699. doi: 10.7860/JCDR/2013/6367.3761. PMID: 24551640, PMCID: PMC3919272.

3) Alhusen JL, Gross D, Hayat MJ, Woods AB, Sharps PW. The influence of maternal - fetal attachment and health practices on neonatal outcomes in low - income, urban women. Res Nurs Health. 2012; 35(2): 112 20. doi: 10.1002/nur.21464. PMID: 22262085, PMCID: PMC3313492.

4) Kliegman RM, Stanton B, Geme JS, Schor NF, Behrman RE. Nelson textbook of pediatrics, Twentieth Edition. Elsevier Health Sciences. 2015.

5) Cortes Castell E, Rizo-Baeza MM, Aguilar Cordero MJ, Rizo-Baeza J, Gil Guillén V. Maternal age as risk factor of prematurity in Spain; Mediterranean area. Nutr Hosp. 2013; 28(5): 1536-40. doi: 10.3305/nh.2013.28.5.6500. PMID: 24160212.

6) Solchany JE. Promoting maternal mental health during pregnancy: Theory, practice and intervention: Nursing Child Assessment Satellite Training. 2001.

7) Lindgren K. Relationships among maternal-fetal attachment, prenatal depression, and health practices in pregnancy. Res Nurs Health. 2001; 24(3): 203-17. doi: 10.1002/nur.1023. PMID: 11526619.

8) Brandon AR, Pitts S, Denton WH, Stringer CA, Evans HM. A history of the theory of prenatal attachment. J Prenat Perinat Psychol Health. 2009; 23(4): 201-22. PMID: 21533008 , PMCID: PMC3083029.

9) Pillitteri A. Maternal \& child health nursing: Care of the childbearing \& childrearing family. Lippincott Williams \& Wilkins. 2010; 1778.

10) Alhusen JL. A literature update on maternal - fetal attachment. J Obstet Gynecol Neonatal Nurs. 2008; 37(3): 315-28. doi: 10.1111/j.1552-6909.2008.00241.x. PMID: 18507602, PMCID: PMC3027206.

11) McFarland J, Salisbury AL, Battle CL, Hawes K, Halloran K, Lester BM. Major depressive disorder during pregnancy and emotional attachment to the fetus. Arch Womens Ment Health. 2011; 14(5): 425-34. doi: 10.1007/s00737.011.0237.z. PMID: 21938509, PMCID: PMC3248759. 
12) Callister, Lynn Clark, Hayman, Laura L. Relationships among maternal-fetal attachment, prenatal depression, and health practices in pregnancy. MCN, American Journal of Maternal Child Nursing. 2002; 27(1): 59. doi: 10.1097/00005721-200201000-00020.

13) Taavoni S, Ahadi M, Ganji T, Hosseini F. Comparison of Maternal Fetal Attachment between Primgravidas and Multigravidas Women with Past History of Fetal or Neonatal Death. Iran Journal of Nursing. 2008; 21(53): 53-61.

14) Cranley MS. Development of a tool for the measurement of maternal attachment during pregnancy. Nurs Res. 1981; 30(5): 281-4. PMID: 6912989.

15) Shakespear K. Centering Pregnancy and traditional prenatal care: A comparison of health practices. All Graduate Theses and Dissertations. 2008; 216.

16) Chanachote S. Spousal Support and Maternal-fetal Attachment in Pregnant Industrial Workers. Mahidol University. 2007; 132.

17) Fogarasi-Grenczer A, Balázs P. The correlation between smoking, environmental tobacco smoke and preterm birth. Orv Hetil. 2012; 153(18): 690-4. doi: 10.1556/OH.2012.29325. PMID: 22547463.

18) Neggers Y, Crowe K. Low birth weight outcomes: why better in Cuba than Alabama? J Am Board Fam Med. 2013; 26(2): 187-95. doi: 10.3122/jabfm.2013.02.120227. PMID: 23471933.

19) Torres-Arreola LP, Constantino-Casas P, Flores-Hernández S, Villa-Barragán JP, Rendón-Macías E. Socioeconomic factors and low birth weight in Mexico. BMC Public health. 2005; 5: 20. doi: 10.1186/1471-2458-5-20. PMID: 15745443, PMCID: PMC554974.

20) Lowdermilk DL, Perry SE, Cashion MC, Alden KR. Study Guide for Maternity and Women's Health Care. Elsevier Health Sciences. 2015. 